\title{
Newly detected open clusters in the Galactic disk using Gaia EDR3 ${ }^{\star}$
}

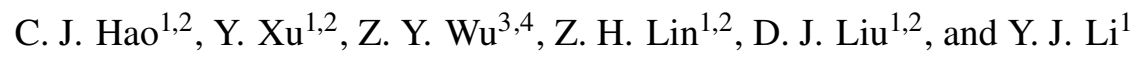 \\ 1 Purple Mountain Observatory, Chinese Academy of Sciences, Nanjing 210023, PR China \\ e-mail: xuye@pmo.ac.cn \\ 2 School of Astronomy and Space Science, University of Science and Technology of China, Hefei 230026, PR China \\ 3 National Astronomical Observatories, Chinese Academy of Sciences, 20A Datun Road, Chaoyang District, Beijing 100101, \\ PR China \\ ${ }^{4}$ School of Astronomy and Space Science, University of Chinese Academy of Sciences, Beijing 101408, PR China
}

Received 12 January 2022 / Accepted 24 January 2022

\begin{abstract}
The astrometric satellite Gaia recently released part of its third data set, which provides a good opportunity to hunt for more open clusters in the Milky Way. In this work, we conduct a blind search for open clusters in the Galactic disk using a sample-based clustering search method with high spatial resolution, which is especially suited to finding hidden targets. In addition to confirming 1930 previously known open clusters and 82 known globular clusters, 704 new stellar clusters are proposed as potential open clusters at Galactic latitudes of $|b| \leq 20^{\circ}$. For each of these new open clusters, we present the coordinates, detailed astrometric parameters, and ages, as well as the radial velocity, if available. Our blind search greatly increases the number of Galactic open clusters as objects of study and shows the incompleteness of the open cluster census across our Galaxy.
\end{abstract}

Key words. Galaxy: stellar content - open clusters and associations: general - methods: data analysis

\section{Introduction}

An open cluster (OC) is a group of stars that formed in the same molecular cloud, so its member stars have approximately the same age and are gravitationally bound to each other. The known ages of OCs cover a wide range, from a few million years to billions of years, making them potentially good tracers for studying the structure and evolution of the Milky Way (e.g., Janes \& Adler 1982; Friel 1995; Buckner \& Froebrich 2014; Hao et al. 2021; Castro-Ginard et al. 2021a; Poggio et al. 2021). In addition, all OCs play an important role in constraining stellar structure and evolutionary models (e.g., Vandenberg 1983; Barnes 2007; Bertelli et al. 2017; Marino et al. 2018). However, recent works have shown that the current catalog of Galactic OCs is still incomplete and, thus, a complete catalog of OCs has yet to be compiled (e.g., Cantat-Gaudin et al. 2018; Castro-Ginard et al. 2018, 2019, 2020; Liu \& Pang 2019).

Accurately searching for Galactic OCs has attracted the interest of many astronomers (e.g., Dias et al. 2002; Kharchenko et al. 2013). Since the publication of the second data release of Gaia (hereafter Gaia DR2; Gaia Collaboration 2016, 2018), studies to detect new OCs and accurately determine membership probability have continued to emerge as an ongoing stream. Based on Gaia DR2, the astrometric parameters and membership probabilities of $\sim 1200$ known OCs presented in previous catalogs ( 3000 objects, e.g., Dias et al. 2002; Kharchenko et al. 2013) were recalculated by Cantat-Gaudin et al. (2018), who also discovered 60 new OCs.

\footnotetext{
* Full Table 1 is only available at the CDS via anonymous ftp to cdsarc.u-strasbg. fr (130.79.128.5) or via http://cdsarc. u-strasbg.fr/viz-bin/cat/J/A+A/660/A4
}

Meanwhile, a machine-learning approach was developed using Gaia Data, with 23 new OCs found in the Tycho-Gaia Astrometric Solution dataset (see Castro-Ginard et al. 2018). Since then, Cantat-Gaudin et al. (2019) found 41 new OCs in the direction of the Perseus Arm. Soon after, 53 new OCs in the direction of the Galactic anti-Centre were detected by Castro-Ginard et al. (2019). Next, by visually inspecting the diagrams of proper motions, Sim et al. (2019) discovered 207 OCs, while Liu \& Pang (2019) reported 2443 stellar clusters, of which 76 were novel OCs of high quality. By applying machine learning in a blind search, Castro-Ginard et al. (2020) identified 582 new OCs in the Galactic disk. Meanwhile, He et al. (2021) found 74 new OCs and Ferreira et al. (2021) discovered 34 new star clusters towards the Galactic bulge. In addition, 41 new OC candidates were reported by Hunt \& Reffert (2021). All of these efforts have greatly improved the amount of Galactic OCs known to astronomers and have also indicated that there are still many unnoticed OC objects that have yet to be cataloged.

Numerous studies also have been conducted to study the astrophysical properties of OCs in Gaia and, simultaneously, to use OCs to unveil the structure of the Milky Way. Through a membership assignment procedure, Cantat-Gaudin \& Anders (2020) derived member stars of 1481 known OCs with Gaia DR2 and investigated their characteristics. In addition, based on Gaia DR2 astrometry, Cantat-Gaudin et al. (2020) compiled a broad and homogeneous OC catalog, in which 1867 clusters have reliable parameters, and they studied the structure and evolution of the Galactic disk using these OCs. Tarricq et al. (2021) computed the kinematic parameters of 1382 OCs with the purpose of revealing the dynamical behaviours of them over time. Meanwhile, a homogeneous sample of fundamental parameters of Galactic open clusters was presented by 
Dias et al. (2021), where they also analyzed the zero-point offset of Gaia data.

The Gaia satellite has released part of its third data set (Gaia Early Data Release 3, hereafter Gaia EDR3), which contains the celestial positions, parallaxes, and proper motions $\left(l, b, \varpi, \mu_{\alpha^{*}}\right.$, and $\left.\mu_{\delta}\right)$, in three photometric bands $\left(G, G_{\mathrm{BP}}\right.$ and $\left.G_{\mathrm{RP}}\right)$ for more than 1.5 billion sources. The astrometric data have been updated significantly relative to DR2, that is, the parallax accuracies have reached 20-30 $\mu$ as (Gaia Collaboration 2020). Moreover, precise radial velocities $(R V \mathrm{~s})$ of seven million stars are also available. Such a vast data store allows us to detect more OCs and enable further studies of the Milky Way.

Based on an unsupervised clustering algorithm, namely, a density-based spatial clustering of applications with noise (DBSCAN, Ester et al. 2020), Castro-Ginard et al. (2018) developed a methodology to find spatial clusters in a five-dimensional (5D) parametric space and then they used an artificial neural network (ANN) approach to automatically distinguish real OCs and statistical clusters. Subsequently, this method was adopted in Gaia DR2 and plenty of new OCs was discovered by them (Castro-Ginard et al. 2019, 2020). Most recently, Castro-Ginard et al. (2021b) reported the discovery of 628 new open star clusters within the Galactic disk again. Up to now, a total of $\sim 1300$ new open clusters were hunted by them, and thus, this result fully demonstrates the power of the algorithms they adopted.

Hao et al. (2020) also adapted the powerful algorithms applied in Castro-Ginard et al. (2018) to detect new OCs (Paper I). Using the OC sample provided in Cantat-Gaudin et al. (2018), Paper I firstly performed a statistical analysis on those known OCs to confirm that there is no need to weigh the 5D parameters (i.e., $l, b, \varpi, \mu_{\alpha^{*}}$ and $\mu_{\delta}$ ) specifically used in the DBSCAN algorithm. Then, Paper I focused on smaller regions, that is, rectangles of $1^{\circ} \times 1^{\circ}$, giving a higher spatial resolution to detect hidden $\mathrm{OCs}$, and normalized the $5 \mathrm{D}$ parameters using $z$-score standardization. Afterwards, inspired by Castro-Ginard et al. (2018), Paper I also used the Gaussian kernel density estimation algorithm (Lampe \& Hauser 2011) and $k$-nearest neighbors algorithm (Altman 1992) to obtain suitable parameters for the DBSCAN algorithm.

The purpose of this work is to blindly search for unnoticed, especially hidden, OCs in the Galactic disk using Gaia EDR3. Here, we amended the sample-based clustering search method used in Paper I to improve its sensitivity to spatial clusters. The OCs were independently hunted in different distance bins and the sizes of the regions being searched varied automatically according to the spatial density of the regions. In addition, to obtain more reliable OC candidates, we propose the stellar clusters as potential OCs based on their kinematics and photometric information.

We organize this paper as follows. Section 2 describes the data used in this work. In Sect. 3, we show how we amended and applied the method in detail. The results of displaying the newly found OCs are presented in Sect. 4. Finally, Sect. 5 summarizes the results obtained in this work.

\section{Data}

The data used in this work are from the Gaia EDR3 catalog (Gaia Collaboration 2020). Most of the known OCs are located in $|b| \leq 20^{\circ}$ ( 96\%, e.g., Dias et al. 2002; Kharchenko et al. 2013; Cantat-Gaudin et al. 2018) and, thus, the expectation of finding OCs in this region is a maximum. Our search focused on the Galactic disk, namely, in the region of $0^{\circ} \leq l \leq 360^{\circ}$ and $-20^{\circ} \leq b \leq 20^{\circ}$. Apart from missing the fraction of bright stars at $G<7$, the Gaia survey is complete in this region (see Gaia Collaboration 2020).

In addition, we only extracted sources brighter than $G=18$, which is the same as the criterion used in Cantat-Gaudin et al. (2018) and Castro-Ginard et al. (2021b). At this magnitude, the median standard uncertainties in the parallaxes are 0.136 mas, and those of the proper motions, $\mu_{\alpha^{*}}$ and $\mu_{\delta}$, are 0.145 and $0.122 \mathrm{mas} \mathrm{yr}^{-1}$, respectively (Lindegren et al. 2021a). As in Liu \& Pang (2019), in order to exclude observational artifacts due to faintness, we also rejected all sources with $\varpi<0$ mas or $\left|\mu_{\alpha^{*}}\right|,\left|\mu_{\delta}\right|>30$ mas yr $^{-1}$. As a result, we obtained a large data set containing 220222696 stars.

\section{Methods}

This section provides a detailed description of the methods used to search for OCs in the Galactic disk and our process for consistently confirming the new OCs. In this work, the samplebased clustering search method used in Paper I has been further amended.

\subsection{Sample division}

Multiple OCs at different distances along the line of sight are superimposed, making it very difficult to accurately decompose them and find the hidden OCs. To solve this puzzle, we suggested in Paper I to make improvements on samples for the purpose of finding hidden OCs by focusing on small spatial regions and considering the effect of distance. Hence, in this work, we independently searched for OCs in different distance bins: $<1 \mathrm{kpc}$, $1-2 \mathrm{kpc}, 2-3 \mathrm{kpc}, 3-4 \mathrm{kpc}, 4-5 \mathrm{kpc}$, and $>5 \mathrm{kpc}$, where each bin includes tens of million stars. Simultaneously, this technique can also reduce the computational procedure.

Another key aspect of the sample-based clustering search method is to decide the spatial sizes of different regions. In our previous work, rectangles of $1^{\circ} \times 1^{\circ}$ were used by Paper I. Here, we have made an improvement on this point, where the sizes varied according to the spatial density of the regions. We still divided the regions into squares, but the sizes of the squares in different distance bins are in the range of $\left[1^{\circ}, 10^{\circ}\right]$, where the higher the density of stars, the smaller the size. This improved measure, which adapts to stellar population densities automatically, is a good solution to the problem of uneven spatial distributions among stars when searching for star clusters. Finally, we yielded about 40000 spatial boxes in the six distance bins.

\subsection{Clustering algorithm}

After preparing the samples (as described in Sect. 3.1) we began to search for spatial overdensity structures using the unsupervised clustering algorithm, DBSCAN. This is a density-based algorithm, so it offers advantages that allow it to find clusters of arbitrary shapes. As the member stars in an OC have common positions and proper motions, we applied DBSCAN in the five-dimensional (5D) space $\left(l, b, \varpi, \mu_{\alpha^{*}}\right.$ and $\left.\mu_{\delta}\right)$. The DBSCAN algorithm defines a cluster based on two parameters: $\epsilon$ and minPts, which are used to describe the closeness of the sample distribution in the neighbourhood. The parameter $\epsilon$ is automatically computed in each sample using a Gaussian kernel density estimation method and the $k$-nearest neighbours algorithm (more details see Paper I). In this work, we were still focused on smaller regions as Paper I to detect unnoticed OCs, namely, the sizes of the squares in different distance bins were set in the 
Table 1. Parameters and errors of some examples of the proposed new OCs ordered by increasing $l$.

\begin{tabular}{|c|c|c|c|c|c|c|c|c|c|c|c|c|c|}
\hline ID & $\begin{array}{c}\alpha \\
{[\mathrm{deg}]}\end{array}$ & $\begin{array}{c}\delta \\
{[\mathrm{deg}]} \\
\end{array}$ & $\begin{array}{c}l \\
\text { [deg] }\end{array}$ & $\begin{array}{c}b \\
\text { [deg] }\end{array}$ & $\begin{array}{l}\theta \\
{[\mathrm{deg}]}\end{array}$ & $\begin{array}{c}\varpi \\
{[\mathrm{mas}]}\end{array}$ & $\begin{array}{c}\mu_{\alpha^{*}} \\
{\left[\mathrm{mas} \mathrm{yr}^{-2}\right]}\end{array}$ & $\begin{array}{c}\mu_{\delta} \\
{\left[\operatorname{mas~yr}^{-2}\right]}\end{array}$ & $\begin{array}{c}\log (\text { age }) \\
{[y r]}\end{array}$ & $\begin{array}{l}A_{\mathrm{G}} \\
{[\mathrm{mag}]}\end{array}$ & $z$ & $\begin{array}{c}V_{r} \\
{\left[\mathrm{~km} \mathrm{~s}^{-2}\right]}\end{array}$ & $\begin{array}{c}N \\
\left(N_{r}\right) \\
\end{array}$ \\
\hline OC-0001 & $7.98 \pm 0.03$ & $-27.85 \pm 0.02$ & $54 \pm 0.02$ & $0.63 \pm 0.02$ & 03 & $5 \pm 0.01$ & $0.75 \pm 0.07$ & $0.12 \pm 0.06$ & $7.40 \pm 0.17$ & 3.22 & 0.028 & $-(-)$ & $17(0)$ \\
\hline OC-0002 & $63.38 \pm 0.07$ & $25.02 \pm 0.05$ & $1.89 \pm 0.05$ & $4.37 \pm 0.06$ & .08 & $2 \pm 0.05$ & 29 & $-0.23 \pm 0.30$ & $8.00 \pm$ & 3.72 & 0.028 & $2.26 \quad(-)$ & $33(1)$ \\
\hline OC-0003 & $70.24 \pm 0.03$ & $24.87 \pm 0.03$ & $5.24 \pm 0.04$ & $-0.88 \pm 0.03$ & 05 & $55 \pm 0.02$ & $1.00 \pm 0.09$ & $-0.19 \pm 0.16$ & $8.80 \pm 0.20$ & 2.56 & 0.028 & $-(-)$ & $16(0)$ \\
\hline OC-0004 & $270.68 \pm 0.02$ & $24.26 \pm 0.03$ & $5.97 \pm 0.02$ & $-0.93 \pm 0.03$ & 0.04 & $0.76 \pm 0.02$ & $1.78 \pm 0.19$ & $-1.88 \pm 0.14$ & $6.60 \pm 0.15$ & 1.38 & 0.016 & $-(-)$ & $17(0)$ \\
\hline OC-0005 & $271.80 \pm 0.03$ & $24.73 \pm 0.03$ & $6.06 \pm 0.04$ & $-2.04 \pm 0.02$ & 0.05 & $0.55 \pm 0.02$ & $0.59 \pm 0.14$ & $-0.79 \pm 0.23$ & $8.10 \pm 0.19$ & 3.46 & 0.028 & $3.36 \quad(-)$ & 20(1) \\
\hline OC-0006 & $271.09 \pm 0.06$ & $-24.37 \pm 0.05$ & $6.06 \pm 0.05$ & $-1.31 \pm 0.06$ & 0.07 & & $1.29 \pm$ & $-2.08 \pm 0.23$ & $6.30 \pm 0.14$ & & & $-(-)$ & $253(0)$ \\
\hline OC-0007 & $268.92 \pm 0.02$ & $-22.67 \pm 0.03$ & $6.54 \pm 0.03$ & $1.26 \pm 0.02$ & 04 & $4 \pm 0.01$ & $-1.32 \pm 0.13$ & $-3.68 \pm 0.11$ & $8.30 \pm 0.19$ & & & $-(-)$ & $23(0)$ \\
\hline OC-0008 & $271.47 \pm 0.02$ & $23.81 \pm 0.02$ & $6.72 \pm 0.02$ & $-1.33 \pm 0.02$ & .03 & $0.52 \pm 0.01$ & $0.27 \pm 0.20$ & $-0.70 \pm 0.13$ & $7.50 \pm 0.17$ & 3.22 & 0.016 & $-(-)$ & $15(0)$ \\
\hline OC-0009 & $271.60 \pm 0.03$ & $-23.25 \pm 0.03$ & $.26 \pm 0.02$ & $-1.16 \pm 0.03$ & 0.04 & $0.36 \pm 0.01$ & $-0.04 \pm 0.17$ & $-1.98 \pm 0.24$ & & 1.78 & 0.016 & $-(-)$ & $15(0)$ \\
\hline OC-0010 & $272.46 \pm 0.01$ & $-23.65 \pm 0.02$ & $7.29=$ & $-2.05 \pm 0.01$ & 0.02 & \pm 0.02 & 19 & $-1.47=$ & 14 & & 0 & $-(-)$ & $16(0)$ \\
\hline OC-0011 & $272.99 \pm 0.05$ & $-22.97 \pm 0.05$ & 0.05 & $-2.15 \pm 0.04$ & .06 & $1 \pm 0.05$ & .17 & .19 & & 3.78 & 0.020 & $11.41 \quad 4.88$ & $289(6)$ \\
\hline OC-0012 & $269.75 \pm 0.03$ & $-20.59 \pm 0.02$ & $8.73 \pm 0.02$ & $1.64 \pm 0.02$ & .03 & $0.32 \pm 0.01$ & $-0.44 \pm 0.13$ & $-1.83 \pm$ & $8.00 \pm 0.18$ & 4.04 & 0.028 & $-(-)$ & $27(0)$ \\
\hline OC-0013 & $271.22 \pm 0.04$ & $-21.36 \pm 0$ & $8.74=$ & $0.06 \pm 0.04$ & 0.07 & $4 \pm 0.02$ & 28 & $-1.03=$ & 7.60 & 4. & 0 & $-(-)$ & $18(0)$ \\
\hline OC-0014 & $271.62 \pm 0.03$ & $21.40 \pm 0.03$ & 03 & $-0.28 \pm 0.03$ & 04 & $9 \pm 0.05$ & .14 & -1.5 & & 4. & 0.0 & $36.95 \quad(-)$ & $79(1)$ \\
\hline OC-0015 & $271.27 \pm 0.05$ & $-21.21 \pm 0.02$ & .03 & $0.10 \pm 0.04$ & .05 & & & -1.2 & & 3.68 & & $-(-)$ & $23(0)$ \\
\hline OC-0016 & $270.23 \pm$ & $-20.21 \pm 0.03$ & $29 \pm$ & $1.44 \pm$ & 0.04 & 01 & $0.19 \pm$ & $-0.11=$ & 6.10 & 2.14 & 0.020 & $-(-)$ & $15(0)$ \\
\hline OC-0017 & $271.95 \pm 0.04$ & $-19.50 \pm 0.06$ & $69 \pm$ & $0.38 \pm$ & 0.07 & $5 \pm 0$ & -0.5 & $-1.96=$ & 23 & & 0. & $-(-)$ & $23(0)$ \\
\hline 18 & $272.03 \pm$ & 2 & 2 & & 3 & & & 24 & & 4 & 26 & $-2.40 \quad(-)$ & $16(1)$ \\
\hline OC-0019 & $274.12 \pm 0.03$ & -20 . & .02 & $-1.72=$ & 0.03 & & & -0. & & 2.28 & 0.0 & $-(-)$ & $15(0)$ \\
\hline OC-0020 & $273.46 \pm 0.02$ & $-18.99 \pm 0.04$ & $.83 \pm 0.04$ & $-0.63 \pm 0.03$ & 005 & 1 & $0.26 \pm$ & -1.64 & 17 & 1.80 & 0 . & $-(-)$ & $15(0)$ \\
\hline OC-0021 & 274 & 4 & 4 & 3 & & & & & & & & $-(-)$ & $33(0)$ \\
\hline OC-0022 & 274. & 18. & 02 & -1.3 & 0.03 & 02 & -0.0 & -1 . & & 1.66 & 0 & $-(-)$ & $17(0)$ \\
\hline OC-0023 & $273.76 \pm 0.02$ & $-17.02 \pm 0.02$ & $.70 \pm 0.02$ & $0.07 \pm 0.02$ & 0.03 & & & -1 . & & 4.04 & & $-(-)$ & $39(0)$ \\
\hline OC-0024 & $274.18 \pm 0.03$ & $-17.01 \pm 0.04$ & $.89 \pm 0.03$ & $8 \pm 0.03$ & & 2 & 14 & -1 & & & & $-(-)$ & $19(0)$ \\
\hline OC-0025 & 3 & & 3 & & & & & & & & & $-(-)$ & $16(0)$ \\
\hline OC-0026 & $274.94 \pm 0.02$ & $-16.54 \pm 0.03$ & $14.66 \pm 0.03$ & $-0.70 \pm 0.02$ & 0.04 & 01 & 15 & $-1.77=$ & 6.50 & 3.98 & 0.016 & $-(-)$ & $16(0)$ \\
\hline OC-0027 & $274.95 \pm 0.03$ & $-15.71 \pm 0.04$ & $15.39 \pm 0.03$ & $-0.32 \pm 0.03$ & 0.05 & $0.44 \pm 0.01$ & $-0.10 \pm 0.10$ & $-2.06 \pm 0.11$ & $7.40 \pm 0.17$ & 3.58 & 0.018 & $-(-)$ & $32(0)$ \\
\hline OC-0028 & $274.64 \pm 0.04$ & $-15.19 \pm 0.04$ & $.71 \pm 0.04$ & & 0.05 & & $-0.53 \pm 0.14$ & & & & 0. & $-6.80(-)$ & $41(1)$ \\
\hline OC-0029 & $275.46 \pm 0.04$ & $-13.89 \pm 0.04$ & $17.23 \pm$ & $0.11 \pm 0.03$ & 0.05 & 05 & -0.6 & -2. & 6.00 & 3.12 & 0.028 & $-(-)$ & $121(0)$ \\
\hline OC-0030 & $273.80 \pm 0.04$ & $-12.56 \pm 0.03$ & $17.64 \pm 0.03$ & $2.16 \pm 0.04$ & 0.05 & $0.61 \pm 0.03$ & $-0.47 \pm 0.24$ & $-0.79 \pm 0.33$ & $7.10 \pm 0.16$ & 2.84 & 0.028 & $-(-)$ & $19(0)$ \\
\hline OC-0031 & $276.29 \pm 0.02$ & $-13.28 \pm 0.02$ & $18.15 \pm 0.02$ & $-0.32 \pm 0.02$ & 0.03 & $0.49 \pm 0.02$ & $-0.45 \pm 0.12$ & $-2.32 \pm 0.10$ & $6.00 \pm 0.14$ & 2.74 & 0.028 & $-(-)$ & $50(0)$ \\
\hline OC-0032 & $277.26 \pm 0.04$ & $-12.71 \pm 0.04$ & $19.08 \pm 0.04$ & $-0.89 \pm 0.03$ & . & & & $-1.64 \pm 0.17$ & & & & $-(-)$ & $25(0)$ \\
\hline OC-0033 & $276.77 \pm 0.03$ & $-12.03 \pm 0.02$ & $19.47 \pm 0.02$ & $-0.16 \pm 0.03$ & 0.04 & $0.37 \pm 0.02$ & $-0.32 \pm 0.09$ & $-2.98 \pm 0.10$ & $7.50 \pm 0.17$ & 3.26 & 0.027 & $37.64 \quad(-)$ & $47(1)$ \\
\hline OC-0034 & $279.00 \pm 0.03$ & $-12.80 \pm 0.02$ & $19.79 \pm 0.02$ & $-2.44 \pm 0.03$ & 0.03 & $0.53 \pm 0.02$ & $0.33 \pm 0.14$ & $-0.51 \pm 0.16$ & $7.60 \pm 0.17$ & 1.98 & 0.028 & $3.65 \quad(-)$ & $19(1)$ \\
\hline OC-0035 & $276.84 \pm 0.03$ & $-10.96 \pm 0.04$ & $20.45 \pm 0.03$ & $0.28 \pm 0.04$ & 0.05 & $0.31 \pm 0.04$ & $-0.43 \pm 0.11$ & $-2.06 \pm 0.14$ & $9.40 \pm 0.22$ & 3.20 & 0.016 & $7.16 \quad 1.73$ & $39(2)$ \\
\hline OC-0036 & $277.18 \pm 0.05$ & $-10.40 \pm 0.05$ & $21.10 \pm 0.05$ & $0.25 \pm 0.05$ & 0.07 & $0.31 \pm 0.01$ & $-0.56 \pm 0.15$ & $-2.41 \pm 0.18$ & $9.80 \pm 0.23$ & 2.28 & 0.017 & $-(-)$ & $33(0)$ \\
\hline OC-0037 & $278.09 \pm 0.03$ & $-10.57 \pm 0.03$ & $21.36 \pm 0.03$ & $-0.63 \pm 0.03$ & 0.04 & $0.52 \pm 0.02$ & $0.65 \pm 0.17$ & $-0.20 \pm 0.12$ & $7.70 \pm 0.18$ & 4.04 & 0.028 & $-10.36(-)$ & $22(1)$ \\
\hline OC-0038 & $279.22 \pm 0.06$ & $-10.91 \pm 0.03$ & $21.57 \pm 0.04$ & $-1.76 \pm 0.05$ & 0.06 & $0.53 \pm 0.01$ & $-0.95 \pm 0.22$ & $-2.72 \pm 0.32$ & $6.90 \pm 0.16$ & 4.04 & 0.028 & $-(-)$ & $20(0)$ \\
\hline OC-0039 & $279.29 \pm 0.02$ & $-10.24 \pm 0.02$ & $22.20 \pm 0.02$ & $-1.52 \pm 0.03$ & 0.03 & & $-1.45 \pm 0.10$ & $-4.33 \pm 0.16$ & & 3.68 & & $-(-)$ & $19(0)$ \\
\hline OC-0040 & $277.95 \pm 0.03$ & $-9.15 \pm 0.05$ & $2.56 \pm 0.04$ & $0.15 \pm 0.03$ & 0.05 & $0.33 \pm 0.01$ & $-0.83 \pm 0.23$ & $-3.18 \pm 0.35$ & $9.80 \pm 0.23$ & 1.94 & 0.017 & $-(-)$ & $19(0)$ \\
\hline
\end{tabular}

Notes. The format of table is the same as in Castro-Ginard et al. $(2020,2021 \mathrm{~b})$. The full table is available at the CDS.

range of $\left[1^{\circ}, 10^{\circ}\right]$. Meanwhile, the parameter minPts is the minimum number of objects within $\epsilon$ that can be considered as a cluster, and since it was been shown in Paper I that many hidden OCs could be hunted with $\operatorname{minPts}=[6,10]$ in small regions, we adopted the same values in this work.

In addition, for stellar clusters located at the borders of the boxes in Sect. 3.1, we took the following measures to dispose of them. If there were two or more clusters with compatible mean parameters within $3 \sigma_{i}$ (where $\sigma$ is the standard deviation) in the 5D parameter space, $i=l, b, \varpi, \mu_{\alpha^{*}}$ and $\mu_{\delta}$, they were considered as substructures of the same star cluster. After further confirmation by visual inspection, we combined these substructures or re-detected the entire cluster. In the following steps, we introduce how we determined if the overdensity structures found with DBSCAN were potentially real OCs or just statistical clusters.

\subsection{Proper-motion dispersion}

As mentioned by Cantat-Gaudin \& Anders (2020), while the apparent proper-motion dispersion of an OC does not constitute an accurate diagnosis of its dynamical state, it can serve as a sufficient empirical basis for distinguishing reasonable OCs from implausible ones. Hence, we filtered the searched star clusters in Sect. 3.2 by their internal proper-motion dispersions. Recently, we found that this method was also adopted in Hunt \& Reffert (2021). In this study, we adopted more rigorous dispersion parameters.

An OC, as a gravitationally bound system, has a small velocity dispersion. The typical dispersion of a globular cluster (GC) is from 5 to $10 \mathrm{~km} \mathrm{~s}^{-1}$ (e.g., Lapenna et al. 2015; Baumgardt \& Hilker 2018). In turn, OCs have smaller dispersions. For example, the study by Mermilliod et al. (2009) showed that the typical dispersion of an OC is $\sim 1 \mathrm{~km} \mathrm{~s}^{-1}$. However, recent studies, using line-of-sight velocities obtained from high-resolution spectroscopy, indicated that an OC has an internal dispersion below $\sim 2 \mathrm{~km} \mathrm{~s}^{-1}$ (e.g., Donati et al. 2014; Cantat-Gaudin et al. 2014; Vereshchagin \& Chupina 2016; Overbeek et al. 2017; Hatzidimitriou et al. 2019). Interestingly, a recent study that focused on the relationship between the total proper-motion dispersions and parallaxes of OCs identified in Gaia DR2 (Cantat-Gaudin \& Anders 2020) showed that the dispersions of most OCs more distant than $\sim 1 \mathrm{kpc}$ (i.e., parallax smaller than 1 mas) are larger than $2 \mathrm{~km} \mathrm{~s}^{-1}$ but below

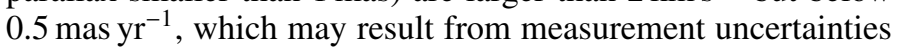



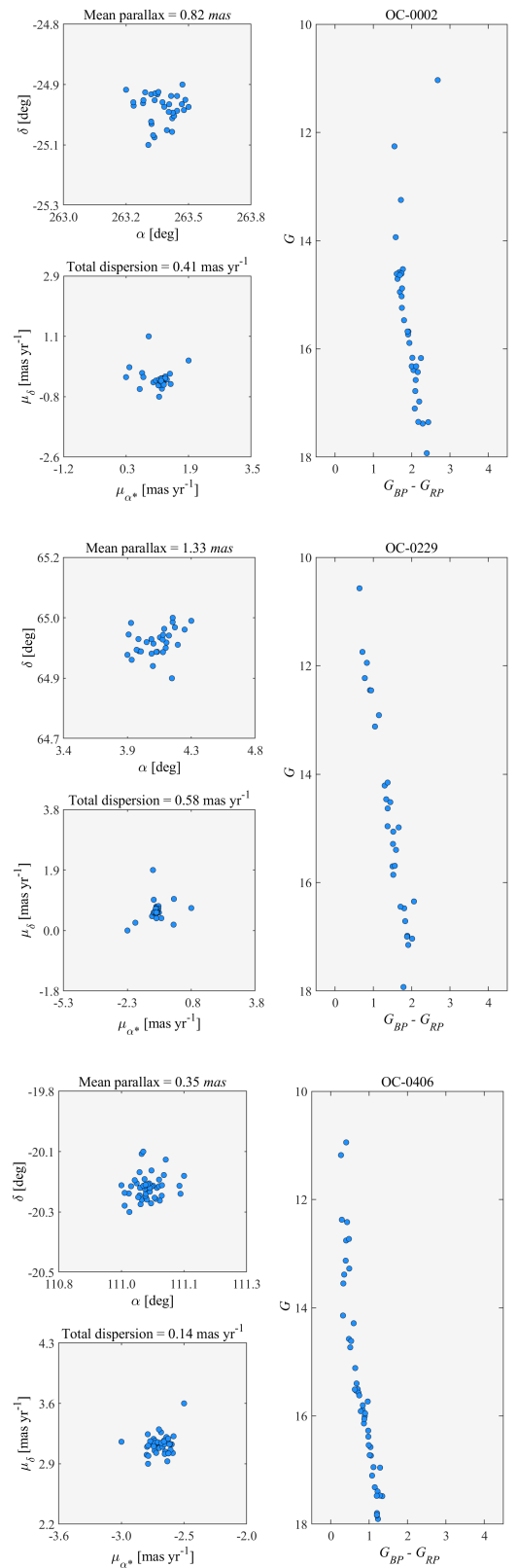
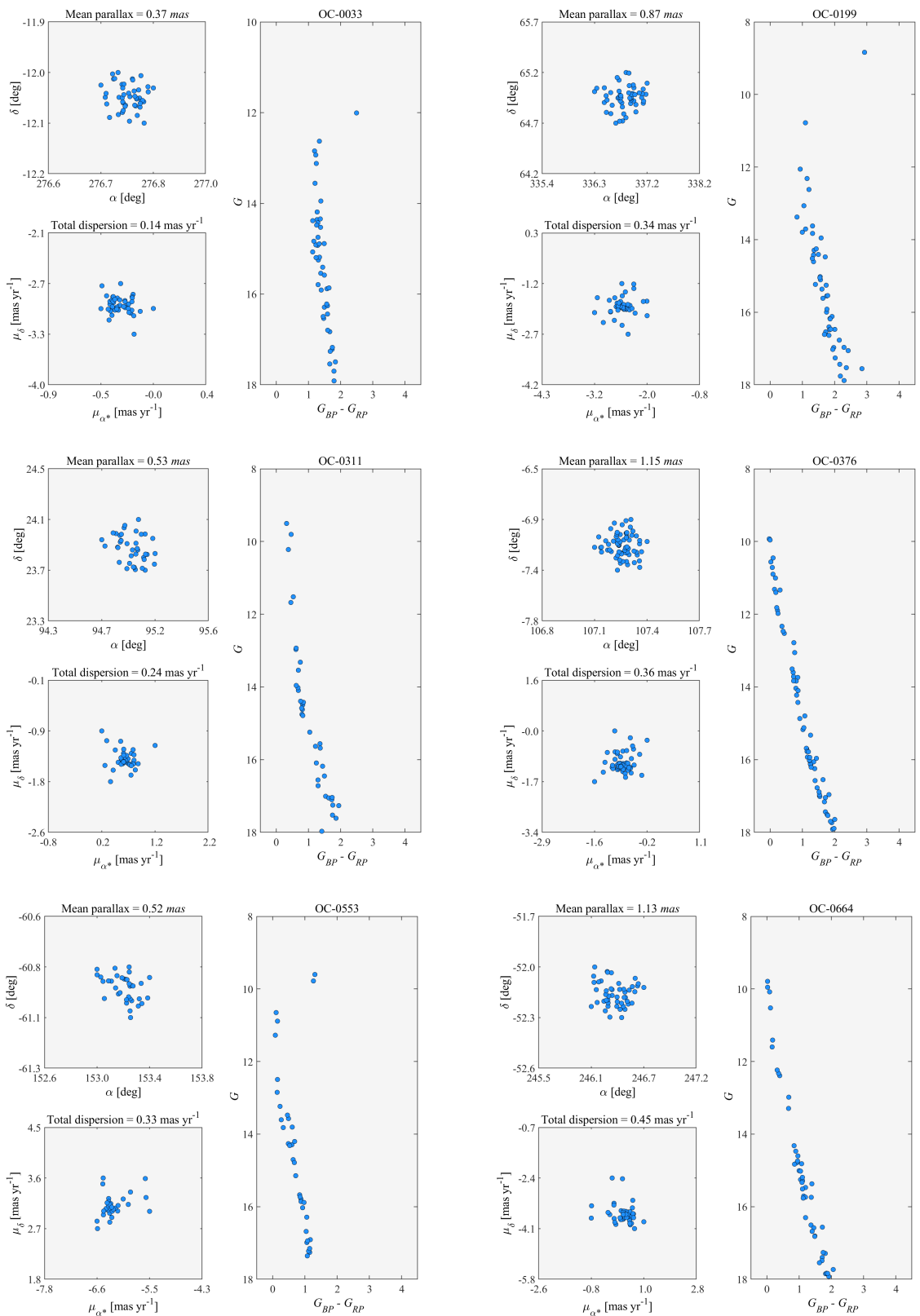

Fig. 1. Examples of class I and class II OCs. For each OC, the columns represent the distributions of the member stars for position in RA and Dec, proper motions in $\mu_{\alpha^{*}}$ and $\mu_{\delta}$, and the CMD in $G$ vs. $G_{\mathrm{BP}}-G_{\mathrm{RP}}$, including its mean parallax and total proper-motion dispersions. Here, the listed OCs are OC-0002, OC-0033, OC-0199, OC-0229, OC-0311, OC-0376 and OC-0406, OC-0553, and OC-0664.

or uncertainties associated with the difficulty of distinguishing between OC member stars and contaminating field stars. Thus, we selected the OC candidates using the proper-motion criterion:

$\sqrt{\sigma_{\mu_{\alpha^{*}}}^{2}+\sigma_{\mu_{\delta}}^{2}} \leq \begin{cases}0.5 \text { mas yr }^{-1} & \text { if } \varpi<1 \text { mas } \\ 2 \sqrt{2} \frac{\varpi}{4.7404} \text { mas yr }^{-1} & \text { if } \varpi \geq 1 \text { mas. }\end{cases}$

\subsection{Confirmation}

For the OC candidates obtained in Sect. 3.3, here we go on to introduce how we proposed them as potentially real OCs. On the one hand, we made references to the characteristics of known OCs described in some previous studies (e.g., Cantat-Gaudin \& Anders 2020; Cantat-Gaudin et al. 2020; Tarricq et al. 2021; Dias et al. 2021); for instance, an OC candidate can be considered as a potentially real OC if it could be shown to clearly pass its members, distance, age, or velocity relative to the field stars, as well as the density of the background stellar distribution, for instance. Furthermore, the member stars of an OC formed in the same molecular cloud, so its color-magnitude diagram (CMD) should follow an empirical isochrone. Hence, we visually inspected the CMDs of the OC candidates found in this work in order to propose the more reliable ones.

In addition, if an OC candidate appeared to be potentially real, we estimated its $R V$ and error as well as its age for further research. As for the weighted standard deviation of its $R V$, we adopted the same equation described by Soubiran et al. (2018):

$\sigma_{R V_{\mathrm{OC}}}^{2}=\frac{\sum_{i} w_{i}}{\left(\sum_{i} w_{i}\right)^{2}-\sum_{i} w_{i}^{2}} \sum_{i} w_{i}\left(R V_{i}-R V_{\mathrm{OC}}\right)^{2}$, 


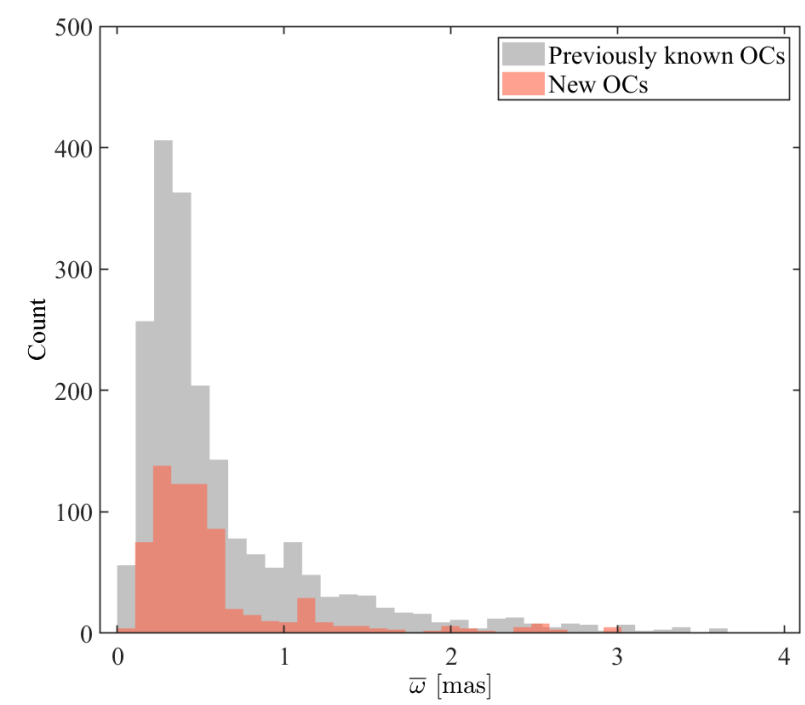

Fig. 2. Parallactic distributions of the newly found OCs (orange) in this work and previously known OCs (grey) from Cantat-Gaudin et al. (2020).

where $R V_{\mathrm{OC}}$ is the mean value of the member stars, $w_{i}=1 / \varepsilon_{i}^{2}$ refers to the individual radial velocity measurements and $\varepsilon_{i}$ is the $R V$ error for star $i$ provided in Gaia EDR3.

Many efforts have been devoted to determining the ages of OCs in Gaia (e.g., Bossini et al. 2019; Cantat-Gaudin et al. 2020). We also determined the ages of the proposed new OCs using the same method adopted in some previous studies (e.g., Liu \& Pang 2019; Hao et al. 2020; He et al. 2021), which is aimed at constructing the best isochrone for the member stars in an OC candidate. The isochrones are derived from the PARSEC library (Bressan et al. 2012) updated for the Gaia DR2 passbands with the photometric calibration of Evans et al. (2018). These isochrones we used contain logarithmic ages (i.e., $\log ($ age/yr)) from 5.92 to 10.13 and metal fractions (z) from 0.015 to 0.029 , as well as a series of isochrones are generated with steps of $\Delta \log ($ age $/ y r)=0.02$ and $\Delta z=0.001$, respectively.

The fitting function is the key to constructing a reliable isochrone for an OC and isochrone fitting pipelines have been produced in several studies (e.g., Perren et al. 2015; Bonatto 2019). As in some previous works, e.g., Liu \& Pang (2019) and He et al. (2021), we used the following fitting function to fit the CMDs for OCs:

$\bar{d}^{2}=\sum_{k=1}^{n}\left|\boldsymbol{x}_{k}-\boldsymbol{x}_{k, n n}\right|^{2} / n$,

where $n$ is the number of member stars in an OC candidate, and $\boldsymbol{x}_{k}=\left[G_{k}+\Delta_{\mathrm{G}}+A_{\mathrm{G}},\left(G_{\mathrm{BP}}-G_{\mathrm{RP}}\right)_{k}+E\left(G_{\mathrm{BP}}-G_{\mathrm{RP}}\right)\right], \boldsymbol{x}_{k, n n}$ is the position of the $k$ th member star and the nearest neighboring point to this star in the isochrone, respectively. For the $\boldsymbol{x}_{k}, \Delta_{\mathrm{G}}$ is the distance modulus, $A_{\mathrm{G}}$ denotes the line-of-sight extinction, and $E\left(G_{\mathrm{BP}}-G_{\mathrm{RP}}\right)$ indicates the reddening in $G$ magnitude of an OC candidate.

The extinction $A_{\mathrm{G}}$ and reddening $E\left(G_{\mathrm{BP}}-G_{\mathrm{RP}}\right)$ of an $\mathrm{OC}$ candidate were corrected as follows. When fitting isochrones, we set the parameter $A_{\mathrm{G}}$ from 0.0 to 4.0 with a step of 0.02 and $E\left(G_{\mathrm{BP}}-G_{\mathrm{RP}}\right)=0.50 A_{\mathrm{G}}$, considering the approximate relation between $A_{\mathrm{G}}$ and $E\left(G_{\mathrm{BP}}-G_{\mathrm{RP}}\right)$ in Gaia (Andrae et al. 2018) as well as an extinction curve of $R_{v}=3.1$ for the Milky Way galaxy (Cardelli et al. 1989; O'Donnell 1994). In addition, parameter $\bar{d}^{2}$ is the mean square distance between OC members and their closest neighboring points in the isochrone. For each OC candidate, we minimized $\bar{d}^{2}$ by calculating the average squares of the distances between member stars and corresponding points in each theoretical isochrone. As mentioned by Liu \& Pang (2019), this method is sensitive to the discrepancy between isochrones and the observational data.

\section{Results}

In total, we detected thousands of stellar clusters in the Galactic disk. After cross-matching with the catalogs from previous works, we obtained 2012 known Galactic stellar clusters, including 1930 OCs and 82 Galactic globular stellar clusters. Among the remaining spatial stellar clusters, we rejected those clusters with large proper-motion dispersions and selected the resulting 910 clusters as OC candidates. Finally, after inspecting the CMDs and other information of these OC candidates, we proposed 704 as potentially real OCs that had previously gone undetected.

\subsection{Cross-matching with known OCs in Gaia}

For all of our detected spatial star clusters, we first crossmatched them with known OC catalogs in Gaia (i.e., Cantat-Gaudin et al. 2018，2019; Castro-Ginard et al. 2018, 2019, 2020, 2021b; Sim et al. 2019; Liu \& Pang 2019; Ferreira et al. 2019, 2020, 2021; Hao et al. 2020; He et al. 2021; Hunt \& Reffert 2021). If a stellar cluster found in this work and a known OC from these catalogs were found to have compatible mean parameters within $3 \sigma_{i}$ in the $5 \mathrm{D}$ astrometric parametric space, $i=l, b, \varpi, \mu_{\alpha^{*}}$ and $\mu_{\delta}$, we considered this star cluster as cross-matched and a visual inspection was also adopted at the same time to confirm this assessment.

Although our aim is to detect hidden OCs in our Galaxy, we still re-detected a large amount of previously known OCs in the Gaia data; for instance: 889 OCs that listed in the catalog ( 1200 known OCs) of Cantat-Gaudin et al. (2018) were refound; we were able to find 37 OCs in the catalog (41 known OCs) of Cantat-Gaudin et al. (2019); 647 OCs reported in Castro-Ginard et al. (2018, 2019, 2020, 2021b) were crossmatched; and so on. In total, the 1759 stellar clusters we obtained were cross-matched with the above catalogs, located in the Galactic latitudes of $|b| \leq 20^{\circ}$, which is comparable with the result (1559) in the recent work of Castro-Ginard et al. (2021b), showing a high re-detection efficiency.

\subsection{Cross-matching with other known OCs}

Before Gaia, Dias et al. (2002) presented a list of OCs based on revised data compiled from previous catalogs and from isolated papers published before, which contained more than 2000 objects. Using the near-infrared photometric data of approximately 470 million objects in 2MASS (Skrutskie et al. 2006), and proper motions from the PPMXL catalog (Röser et al. 2010), Kharchenko et al. (2013) completed a survey of all previously known OCs, called the Milky Way Star Clusters (MWSC). This catalog lists 3006 objects, including known OCs and OC candidates (2469), GCs, associations, and asterisms.

After completing the step in Sect. 4.1, we cross-matched the remaining clusters with the above two catalogs using the following criteria: if a star cluster falls within a circle of $0.5^{\circ}$ radii (Castro-Ginard et al. 2019, 2020) of one object listed in these two catalogs, we selected it. Then, if their mean proper 


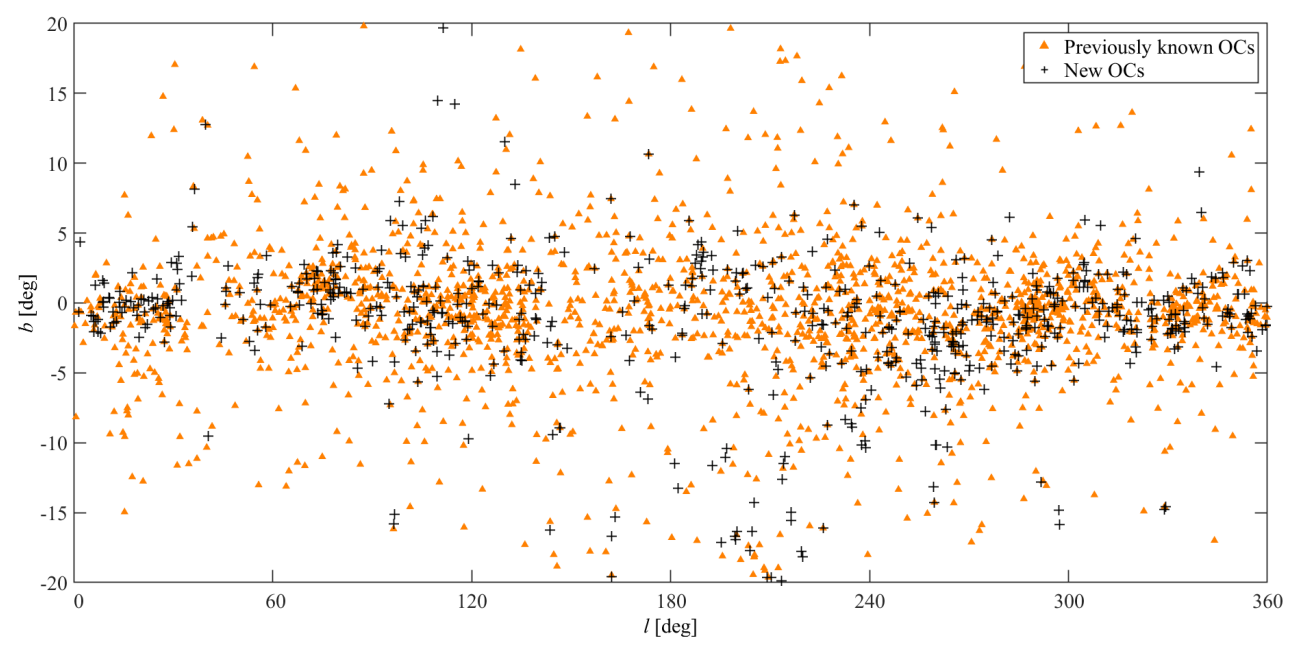

Fig. 3. Distributions of $l$ versus $b$ of the newly found OCs and previously known OCs, where the black plus signs represent the new OCs and the orange triangles represent the known OCs compiled in Cantat-Gaudin et al. (2020).

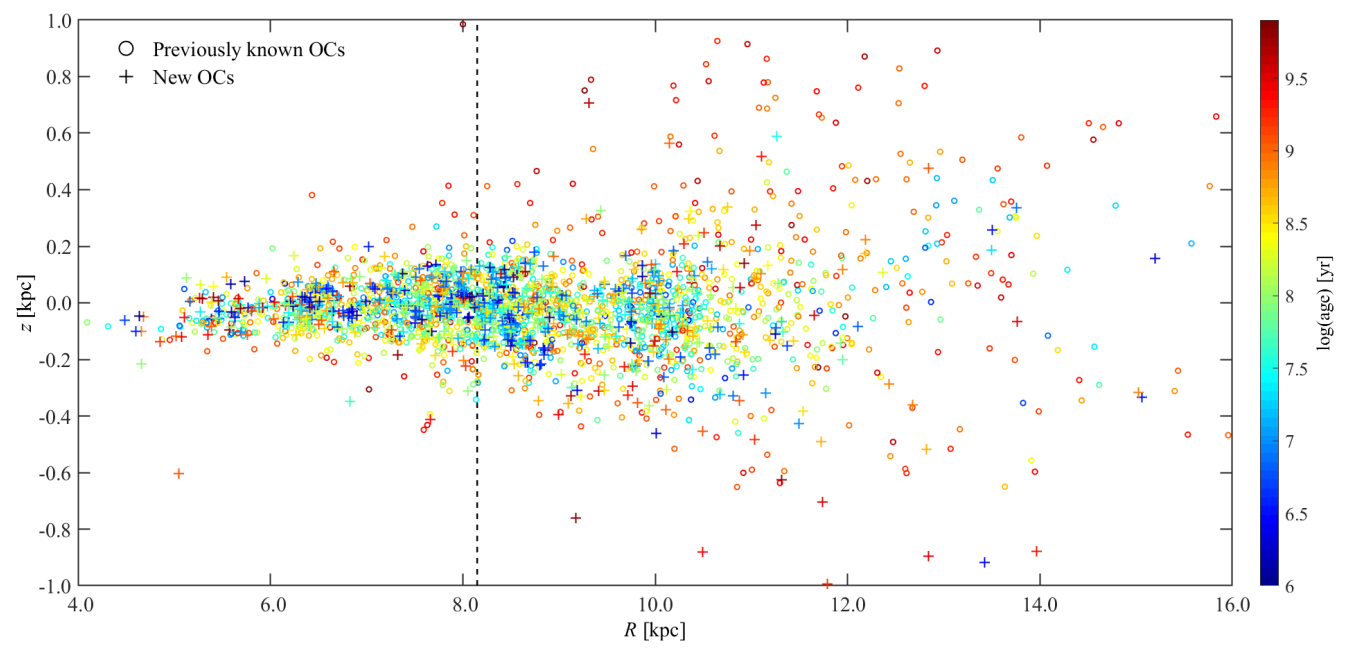

Fig. 4. Distributions of $R$ versus $z$ of the newly found OCs and previously known OCs, and the same as Fig. 3, the plus signs represent the new OCs and the triangles represent the known OCs (Cantat-Gaudin et al. 2020), but the ages of the OCs are color-coded here. The Solar circle (black dashed) is at $8.15 \mathrm{kpc}$ (Reid et al. 2019).

motions were within $3 \sigma_{i}$ of the cluster found in this work and their distances were compatible, we considered this star cluster as being matched. The catalogs in Dias et al. (2002) and Kharchenko et al. (2013) do not provide the mean parallax and associated uncertainty for each OC but an estimation of the distance instead, so the criterion is that the known OCs are within the distance errors of our detected clusters. The distance of our detected cluster is the inverse of the mean parallax of member stars (Castro-Ginard et al. 2020), and the assumed errors come from three times the standard deviation of parallax. Although most of the OCs in these two catalogs had already been taken into account by the cross-matching of our star clusters with the sample of Cantat-Gaudin et al. (2018), we still found 120 known OCs in the MWSC catalog and 71 known OCs in the catalog of Dias et al. (2002), along with 20 OCs that are repetitive.

\subsection{Cross-matching with known GCs}

In order to cross-match with known GCs more accurately, we investigated the GC catalog reported by Baumgardt et al. (2019), who presented the mean proper motions and space velocities of 154 Galactic GCs using a combination of Gaia DR2 proper motions and ground-based line-of-sight velocities. Finally, we found that 82 star clusters we obtained were cross-matched with the GCs listed by Baumgardt et al. (2019), and the proper motions and $R V$ s provided by Gaia EDR3 are consistent with those in the catalog.

There are 106 GCs presented by Baumgardt et al. (2019) located at the Galactic latitude of $|b| \leq 20^{\circ}$, and, thus, the proportion of GCs detected here is $\sim 80 \%$. Each GC found here contains thousands of stars, and the detection of Galactic GCs is a good test of our proposed method. Meanwhile, for the remaining $\sim 20 \%$ of GCs that were not detected by us, their non-detection might be the result of their far distances make their member stars being too faint.

\subsection{New OCs}

After cross-matching with the known Galactic OC and GC catalogs, there were many star clusters remaining whose cluster nature was yet to be determined. Among these clusters, 910 ones met the proper-motion criterion described in Sect. 3.3. These 


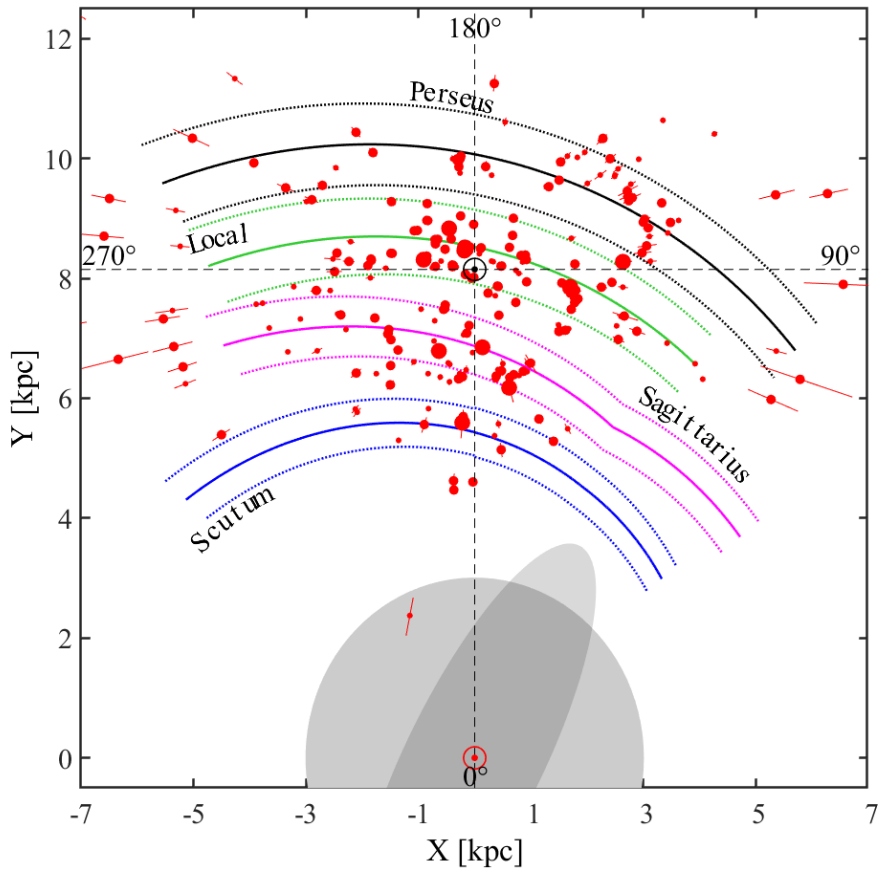

Fig. 5. Locations of the newly found OCs younger than 20 million years projected onto the Galactic plane, including their $1 \sigma$ distance uncertainties. The sizes are proportional to the number of members of each cluster. The solid and dashed curved lines denote the arm centre and widths (i.e., which enclose $90 \%$ of the masers), respectively, fitted by Reid et al. (2019) from their parallax data of Very Long Baseline Interferometry masers. The Galactic Centre (red symbol) is at $(0,0) \mathrm{kpc}$ and the Sun (black symbol) is at $(0,8.15) \mathrm{kpc}$.

910 star clusters were considered as potential OC candidates. We visually inspected the features of these star clusters, as described in Sect 3.4. After this inspection, 704 OC candidates were proposed to be potentially real OCs.

Based on the same format as the table presented in previous works (e.g., Castro-Ginard et al. 2020, 2021b), we show the mean parameters and standard deviations (i.e., $1 \sigma$ errors) of each OC candidate in Table 1, including its right ascension (RA), declination (Dec), $l, b$, parallax $(\varpi)$, proper motions $\left(\mu_{\alpha^{*}}\right.$ and $\left.\mu_{\delta}\right)$, age $(\log (t)), R V\left(V_{r}\right)$, number of member stars $(N)$, and its apparent angular size, which was computed by $\theta=\sqrt{\sigma_{l}^{2}+\sigma_{b}^{2}}$ following Castro-Ginard et al. (2020). The full version of Table 1 can be found online at the CDS, including the table of member stars of OCs. For our newly found OCs, Fig. 1 shows some examples of the distributions of the member stars in astrometric space and their CMDs. It is obvious that the OCs we proposed typically show a high concentration of member stars in all five astrometric parameters, especially the proper motions, and their member stars in the CMDs are almost concentrated on potentially theoretical isochrones.

\subsection{Characteristics of the new OCs}

As shown in Fig. 2, we made a parallactic comparison between the newly found OCs and the previously known OCs in Cantat-Gaudin et al. (2020), which shows a high level of consistency. For the newly found OCs, the mean parallaxes of vast majority $(99.3 \%$ ) range from 0.06 to 3.00 mas, which yield distances from $\sim 330 \mathrm{pc}$ to $\sim 16 \mathrm{kpc}$ when estimating the distance as the inverse of the mean parallax. However, we note that this method is not ideal as it ignores the parallax uncertainties (e.g., Lindegren et al. 2021b). In addition, $\sim 15 \%$ of the OCs are closer than $1 \mathrm{kpc}, \sim 34 \%$ are located between 1 and $2 \mathrm{kpc}, \sim 37 \%$ between 2 and $4 \mathrm{kpc}$, and $\sim 14 \%$ of the OCs are further than $4 \mathrm{kpc}$. Among the newly found OCs, 214 of them have stars with $R V \mathrm{~s}$ in Gaia EDR3, of which 71 OCs contain more than two stars with $R V \mathrm{~s}$.

For the newly found OCs, $\sim 85 \%$ are located at Galactic latitudes of $|b| \leq 5^{\circ}, \sim 9 \%$ are located in $5^{\circ}<|b| \leq 10^{\circ}$ and only $\sim 6 \%$ are located at Galactic latitudes $|b|>10^{\circ}$, showing they are concentrated in the Galactic disk. The distributions of the new OCs in the Galactic plane are shown in Fig. 3, which indicates that the distribution of the new OCs follows a particularly similar distribution to the previously reported ones. Figure 4 presents the distribution of the new OCs and known OCs in the galactocentric radius $(R)$ and altitude $(z)$ above the Galactic middle plane, showing they are very consistent, and this result is also concordant with Castro-Ginard et al. (2021b). Hence, all of the above distributions of our newly found hidden OCs significantly densify the distribution constructed by previously known OCs.

The ages of the proposed OCs are color-coded in Fig. 4, which reveals the consistency of the age estimations with the previously known OC population compiled by Cantat-Gaudin et al. (2020). Besides, it can be seen that these new OCs gradually migrated further from the Galactic disk as they age, which has been mentioned by Hao et al. (2021).

The distribution of the 299 newly found OCs with ages younger than 20 million years projected on to the Galactic plane is shown in Fig. 5, where the spiral arms defined by masers associated with high-mass star formation regions with Very Long Baseline Interferometry parallax measurements (Reid et al. 2019) are also displayed for comparison. More than $70 \%$ of the young OCs are located in the spiral arms outlined by high-mass star formation region masers or bright O-B type stars (Xu et al. 2018, 2021), especially the more populous ones and the distribution of young OCs is similar to those of O-B type stars in Gaia. Hence, our result also confirmed that the young OCs are good tracers of the Galactic spiral structure, as described in some recent works (e.g., Hao et al. 2021; Castro-Ginard et al. 2021a; Poggio et al. 2021).

\section{Conclusion}

Our proposed methodology, an amended version of samplebased clustering search method, which possess high spatial resolution, combined with the high-precision astrometric and photometric data in Gaia EDR3, allowed us to find many previously unnoticed and hidden OCs in the 5D astrometric space $\left(l, b, \varpi, \mu_{\alpha^{*}}\right.$, and $\left.\mu_{\delta}\right)$. In this work, the detection of over-density structures was performed in different distance bins as well as small spatial regions and multiple sample sizes were used for regions with different stellar densities, which do contribute to search for undetectable OCs in the Galactic disk. Then, the stellar clusters were reduced and confirmed as potentially real OCs based on their distributions, proper-motion dispersions, CMDs and $R V \mathrm{~s}$, if available.

Our blind search of the Galactic disk produced 1930 known OCs and 82 known GCs, as well as 704 potentially new OCs. The new OCs have greatly improved the completeness of the Galactic OC census, and they commendably extend the distribution of known OCs. Indeed, the high re-detection effectiveness for GCs also proves the reliability of our proposed method. 
Acknowledgements. We appreciate the anonymous referee for the instructive comments which help us to improve the paper. This work was funded by NSFC Grants 11933011, 11873019, 11673066 and 11988101, and the Key Laboratory for Radio Astronomy. The authors thank Dr. Castro-Ginard for kindly providing the data of their newly found open clusters in Castro-Ginard et al. (2021b). Y.J.L. thanks support from the Natural Science Foundation of Jiangsu Province (grant number BK20210999). This work has made use of data from the European Space Agency (ESA) mission Gaia (https://www . cosmos.esa.int/gaia), processed by the Gaia Data Processing and Analysis Consortium (DPAC, https://www.cosmos.esa.int/web/gaia/dpac/ consortium). Funding for the DPAC has been provided by national institutions, in particular the institutions participating in the Gaia Multilateral Agreement.

\section{References}

Altman, N. S. 1992, BAN, 46, 175

Andrae, R., Fouesneau, M., Creevey, O., et al. 2018, A\&A, 616, A8

Barnes, Sydney A. 2007, ApJ, 669, 1167

Baumgardt, H., \& Hilker, M. 2018, MNRAS, 478, 1520

Baumgardt, H., Hilker, M., Sollima, A., \& Bellini, A. 2019, MNRAS, 482, 5138

Bertelli, M. C., Salaris, M., Pasquali, A., \& Grebel, E. K. 2017, MNRAS, 466, 2161

Bonatto, C. 2019, MNRAS, 483, 2758

Bossini, D., Vallenari, A., Bragaglia, A., et al. 2019, A\&A, 623, A108

Bressan, A., Marigo, P., Girardi, L., et al. 2012, MNRAS, 427, 127

Buckner, A. S. M., \& Froebrich, D. 2014, MNRAS, 444, 290

Cantat-Gaudin, T., \& Anders, F. 2020, A\&A, 633, A99

Cantat-Gaudin, T., Vallenari, A., Zaggia, S., et al. 2014, A\&A, 569, A17

Cantat-Gaudin, T., Jordi, C., Vallenari, A., et al. 2018, A\&A, 618, A93

Cantat-Gaudin, T., Krone-Martins, A., Sedaghat, N., et al. 2019, A\&A, 624, A126

Cantat-Gaudin, T., Anders, F., Castro-Ginard, A., et al. 2020, A\&A, 640, A1

Cardelli, J. A., Clayton, G. C., \& Mathis, J. S. 1989, ApJ, 345, 245

Castro-Ginard, A., Jordi, C., Luri, X., et al. 2018, A\&A, 618, A59

Castro-Ginard, A., Jordi, C., Luri, X., Cantat-Gaudin, T., \& Balaguer-Nunez, L. 2019, A\&A, 627, A35

Castro-Ginard, A., Jordi, C., Luri, X., et al. 2020, A\&A, 635, A45

Castro-Ginard, A., McMillan, P. J., Luri, X., et al. 2021a, A\&A, 652, A162

Castro-Ginard, A., Jordi, C., Luri, X., et al. 2021b, A\&A, submitted, [arXiv:2111.01819v1]

Dias, W. S., Alessi, B. S., Moitinho, A., \& Lépine, J. R. D. 2002, A\&A, 389, 871

Dias, W. S., Monteiro, H., Moitinho, A., et al. 2021, MNRAS, 504, 356

Donati, P., Cantat-Gaudin, T., Bragaglia, A., et al. 2014, A\&A, 561, A94
Ester, M., Kriegel, H. P., Sander, J., \& Xu, X. 2020, 2nd International Conference on Knowledge Discovery and Data Mining (AAAI Press), 96, 226

Evans, D. W., Riello, M., De Angeli, F., et al. 2018, A\&A, 616, A4

Ferreira, A. F., Santos, J. F. C., Corradi, W. J. B., Maia, F. F. S., \& Angelo, M. S. 2019, MNRAS, 483, 5508

Ferreira, A. F., Corradi, W. J. B., Maia, F. F. S., Angelo, M. S., \& Santos, J. F. C. 2020, MNRAS, 496, 2021

Ferreira, A. F., Corradi, W. J. B., Maia, F. F. S., Angelo, M. S., \& Santos, J. F. C. 2021, MNRAS, 502, L90

Friel, E. D. 1995, ARA\&A, 33, 381

Gaia Collaboration (Prusti, T., et al.) 2016, A\&A, 595, A1

Gaia Collaboration (Brown, A. G. A., et al.) 2018, A\&A, 616, A1

Gaia Collaboration (Brown, A. G. A., et al.) 2020, A\&A, 649, A1

Hao, C. J., Xu, Y., Wu, Z. Y., et al. 2020, PASP, 132, 034502

Hao, C. J., Xu, Y., Hou, L. G., et al. 2021, A\&A, 652, A102

Hatzidimitriou, D., Held, E. V., Tognelli, E., et al. 2019, A\&A, 626, A90

He, Z. H., Xu, Y., Hao, C. J., Wu, Z. Y., \& Li, J. J. 2021, Res. Astron. Astrophys., 21, 093

Hunt, E. L., \& Reffert, S. 2021, A\&A, 646, A104

Janes, K., \& Adler, D. 1982, ApJS, 49, 425

Kharchenko, N. V., Piskunov, A. E., Schilbach, E., Röser, S., \& Scholz, R.-D. 2013, A\&A, 558, A53

Lampe, O. D., \& Hauser, H. 2011, 2011 IEEE Pacific Visualization Symp (Piscataway, NJ: IEEE), 171

Lapenna, E., Origlia, L., Mucciarelli, A., et al. 2015, ApJ, 798, 23

Lindegren, L., Klioner, S. A., Hernández, J., et al. 2021a, A\&A, 649, A2

Lindegren, L., Bastian, U., Biermann, M., et al. 2021b, A\&A, 649, A4

Liu, L., \& Pang, X. 2019, ApJS, 245, 32

Marino, A. F., Milone, A. P., Casagrande, L., et al. 2018, ApJ, 863, L33

Mermilliod, J. C., Mayor, M., \& Udry, S. 2009, A\&A, 498, 949

O’Donnell, J. E. 1994, ApJ, 422, 158

Overbeek, J. C., Friel, E. D., Donati, P., et al. 2017, A\&A, 598, A68

Perren, G. I., Vázquez, R. A., \& Piatti, A. E. 2015, A\&A, 576, A6

Poggio, E., Drimmel, R., Cantat-Gaudin, T., et al. 2021, A\&A, 651, A104

Röser, S., Demleitner, M., \& Schilbach, E. 2010, AJ, 139, 2440

Reid, M. J., Menten, K. M., Brunthaler, A., et al. 2019, ApJ, 885, 131

Sim, G., Lee, S. H., Ann, H. B., \& Kim, S. 2019, J. Korean Astron. Soc., 52, 145

Skrutskie, M. F., Cutri, R. M., Stiening, R., et al. 2006, AJ, 131, 1163

Soubiran, C., Cantat-Gaudin, T., Romero-Gómez, M., et al. 2018, A\&A, 619, A 155

Tarricq, Y., Soubiran, C., Casamiquela, L., et al. 2021, A\&A, 647, A19

Vandenberg, E. D. 1983, ApJS, 51, 29

Vereshchagin, S. V., \& Chupina, N. V. 2016, Balt. Astron., 25, 432

Xu, Y., Bian, S. B., Reid, M. J., et al. 2018, A\&A, 616, L15

Xu, Y., Hou, L. G., Bian, S. B., et al. 2021, A\&A, 645, L8 